\title{
Energy Harvesting Technique for Efficient Wireless Cognitive Sensor Networks Based on SWI PT Game Theory
}

\author{
Fadhil Mukhlif ${ }^{1, *}$, Kamarul Ariffin Bin Noordin ${ }^{2, *}$, Omar B. Abdulghafoor ${ }^{3}$ \\ ${ }^{1,2}$ Department of Electrical Engineering, Faculty of Engineering, University of Malaya, Kuala Lumpur, Malaysia \\ [e-mail: fadhil.researcher@gmail.com], [e-mail: Kamarul@um.edu.my] \\ ${ }^{3}$ Electronic and Telecommunication Department, College of Engineering, the American University of Kurdistan, \\ Iraq \\ [e-mail: omar.omarghafoor@gmail.com] \\ * Corresponding authors: Kamarul Ariffin Bin Noordin; Fadhil Mukhlif
}

Received February 10, 2020; revised April 1, 2020; accepted May 4, 2020;

published June 30, 2020

\begin{abstract}
The growing demand to make wireless data services 5G compatible has necessitated the development of an energy-efficient approach for an effective new wireless environment. In this paper, we first propose a cognitive sensor node (CSN) based game theory for deriving energy via a primary user-transmitted radio frequency signal. Cognitive users' time was segmented into three phases based on a time switching protocol: energy harvest, spectrum sensing and data transmission. The proposed model chooses the optimal energy-harvesting phase as the effected factor. We further propose a distributed energy-harvesting model as a utility function via pricing techniques. The model is a non-cooperative game where players can increase their net benefit in a selfish manner. Here, the price is described as a function pertaining to transmit power, which proves that the proposed energy harvest game includes Nash Equilibrium and is also unique. The best response algorithm is used to achieve the green connection between players. As a result, the results obtained from the proposed model and algorithm show the advantages as well as the effectiveness of the proposed study. Moreover, energy consumption was reduced significantly (12\%) compared to the benchmark algorithm because the proposed algorithm succeeded in delivering energy in micro which is much better compared to previous studies. Considering the reduction and improvement in power consumption, we could say the proposed model is suitable for the next wireless environment represented in 5G.
\end{abstract}

Keywords: cognitive sensor network (CSN), WSN, SWIPT, time switching (TS), power control, green communication, game theory, $5 \mathrm{G}$ network 


\section{Introduction}

In the future, humans will witness a linked community. The Internet of Things (IoT), as well as smart, assimilated systems with built-in sensors, cloud radio access networks (C-RAN) and in-house sensing networks, would transform the manner in which individuals live. [1] [2] and [3]. Furthermore, such a networked system should enhance energy efficacy, cost and spectrum usage. It should also deliver improved scalability for dealing with the increasing number of linked devices. Considering the idea of a connected ecosystem in recent networks, the general technical objective is to present a model which would bolster high energy with spectral efficacy [4]. A wireless sensor network (WSN) comprises multiple sensor nodes that have drawn significant attention and uses of late [5]. For instance, for comprehending our environment better and turning it into a niftier state, a type of media for connecting the cyber space with the actual world ought to be devised. Then, the idea of IoT emerged, with the universal wireless sensor networks (WSNs) playing the role of a key fundamental technology to bolster IoT. Wireless networks find applications not only in mobile cloud computing (MCC) but also in the healthcare sector, wherein physiological activities and human actions can be monitored continuously with the help of wearable implantable biosensors [6]. Moreover, applications of WSN have become common even in the unlicensed industrial, medical and scientific bands, which are quickly becoming overcrowded each day, as these bands allow for sharing information with other wireless applications [7] [8]. The licensed bands are not utilised efficiently, thereby causing a grave imbalance in the usage of radio spectrum. As a result, not only the available spectrum must be used efficiently but also there is a need to effectively manage spectrum when developing wireless applications, and in this regard, the concept pertaining to cognitive radio (CR) was introduced [9]. CR can be employed to use the available licensed spectrum. This can be done by reconfiguring its features and by exploiting its cognitive capabilities, which allows for unlicensed secondary users (SUs) to utilise part of the licensed frequency range that has been underutilised and will not interfere in the activities pertaining to the licensed primary users (PUs) [10]. A wireless cognitive sensor network (CSN) can be defined as a WSN that possesses CR capabilities. Moreover, with regards to the opportunistic use of the licensed spectrum, the unlicensed band can also be used by SUs without disrupting communication amongst PUs. Hence, CSN entails the basic attributes of CR networks [11, 12].

From the perspective of wireless infrastructure, two key kinds of energy harvesting have surfaced: radio frequency and environmental [13] and [14]. Radio frequency of energy harvesting presents captivating prospects, with the potential of reducing the chances occurring in the wireless power source. The idea encompasses mingling energy harvesting linked to wireless power transmission methods $[15,16]$. The technique facilitates energy sharing between network nodes [17], which in turn extends nodes' lifetime when the energy of battery is low [18] [19]. Energy signals are superimposed within other signals of regular communication, which takes this approach to the next level and in turn allows for 'simultaneous wireless information and power transfer (SWIPT)' [20] [21].

An effective power control algorithm is required in CSNs to reduce their interference during spectrum sharing. It is also required to preserve the desired signal-to-interference ratio (SIR) and to extend CSN devices' lifetime. However, game and economic theories have been published as an alternative to the issues of energy control in wireless data networks, as the choice of service depends on the functionality of the tool. The focus in game theory is to 
design utility functions where the game has a physical connotation and its result is not important [22].

Energy control algorithms can be developed in many ways in terms of instrument performance and price. According to some researchers, utilities are the difference between price and utility functions, and consequently consumers generally tend to upgrade these tools in a selfish way. In such a scenario, the utility function should be half, and the optimum point within the practical parameters, such as maximum and minimum power, is also selected, depending on the behaviour of the other user. The utility function of this type is related to energy efficiency, as it has a physical meaning connected to the information it receives successfully and can be calculated based on the bits per joule of energy cost. To obtain the required SIR in the network, all users adjust the transmission power, which is not demarcated clearly in the utility function but relies on the effectiveness of function expectation [23].

\subsection{Contributions}

There are many techniques that can be adopted to allocate power and resources to users in a wireless network. For example, evolutionary algorithms such as particle swarm optimisation (PSO) and genetic algorithm (GA), as highlighted in [24, 25], provide a good and direct solution to the mix-integer optimization problem. However, they suffer from poor convergence to steady point. Hence, these types of algorithms are not that much efficient in designing power allocation model in heterogeneous wireless networks.

Pricing-based non-cooperative game theory is one of the significant techniques that have been adopted in resource allocation problems in wireless networks for the following reasons: (i) Pricing can provide an economical NE by directing selfish players to a more costeffective operating point as highlighted in [26]; (ii) Pricing-based non-cooperative game can be adopted as a punishment for those players that generate interference to the owner of the spectrum, PUs, and hence provide a convenient coexistence between cognitive users and primary users. However, the use of pricing-based non-cooperative game only resulted in slow convergence to the NE, as can be observed in [23].

Our proposed algorithm has been compared with the algorithms proposed in [23], [27-29]. Authors in [23] developed an approach for distributed power control in CRN based on utility-pricing technique. The utility function is chosen as a QoS of CR user based on pricing technique and a distributed power control algorithm is developed as a non-cooperative game in which users maximise their net utility. The pricing function is defined as a real function of transmit power to increase pricing charge of the remote CR users, and the existence and uniqueness of the NE have been proved analytically. However, the convergence of the proposed algorithm to the stable point, NE, is slow.

The authors in [27] propose a modified power control algorithm based on non-cooperative game theory. Furthermore, to improve the accuracy of the proposed optimization, the shuffled frog leaping algorithm (SFLA) is modified by adopting the basic ideas of artificial fish (AF). However, the convergence of the proposed algorithm to the NE is even slower than that in [23].

In [28], the authors propose an efficient power control based on the non-cooperative game theory. The pricing technique is proposed to design non-linear pricing function to add some 
improvement to the Pareto region in the non-cooperative power control game. However, the uniqueness of the NE is ignored in the proposed algorithm.

Finally, a price-based power control algorithm in a CRN is studied in [29]. The proposed utility function considered the throughput fairness among cognitive wireless nodes, where the SINR information for CRs is used as reference for the pricing punishment parameter setting. However, the uniqueness of the NE is ignored in this research, which makes the proposed algorithm unsuitable for heterogeneous networks.

In this research, distinct from the existing studies that propose similar algorithms, we assess the power allocation issue associated with distributed cognitive sensors (CSs) in SWIPT, which include multiple coexisting sensors set at the same frequency band within a communication system. Our main interest lies in a non-cooperative method, because the future distributed multiple cognitive sensors system could face certain implementation challenges, wherein these sensors may fail to cooperate. Thus, autonomous distributed power allocation techniques need to be considered, which offer a key benefit of avoiding energy consumption usually seen in centralised policies that need substantial information exchange amongst sensors. Here, the primary aim would be to ensure a predefined SIR requirement pertaining to targeted sensors, while at the same time, keeping power consumption to the minimum for every sensor with effective optimisation of transmission power allocation. Moreover, the smooth and fast convergence of the designed algorithm to the NW is another advantage of a combination of SWIPT and pricing techniques. The key contributions of this work are stated below:

1. We introduce a utility function comprising a targeted SIR-weighted exponent and the required signal as well as the pricing function that includes a power function pertaining to the transmit power of CSs. Some of the key features of the proposed energy-harvesting scheme are as follows:

a) It allows for conserving the needed QoS related to the efficiency of all CSs, with a considerable decrease in signal-to-noise ratio.

b) It practically applies the algorithm in a distributive manner and does not even need additional information.

c) It ensures considerable reduction as well as power allocation enhancement for all CSs.

d) It facilitates Nash equilibrium quick convergence.

2. The novelty of the proposed scheme is that it can describe how energy-harvesting time can be selected for balancing the energy renewal as well as energy consumption in CS.

3. The transmission time pertaining to cognitive users can be segmented into three parts: energy-harvesting phase, spectrum-sensing phase and data transmission phase to reply to the time switching (TS).

4. The pricing and utility functions are important to allow each CS to select transmitting power efficiency. The model also allows for guiding the closest CSs towards the base station so that QoS requirement could be addressed cost effectively. It is also easy to guide CSs away from the base station, which helps address the quality of service (QoS) prerequisites and the high expenses for reducing collisions. 
5. Evaluation is done for the problem pertaining to non-cooperative power control challenge for the existing sensors by taking pricing into account. The proposed game model also achieves Nash equilibrium in games of a non-cooperative feature, showing its uniqueness.

6. This study provides a new illustration that offers a better understanding to the concept of NE, instead of a mathematical approach of NE.

7. An iterative power allocation algorithm is developed that allows for fast convergence and is associated with low computational complexity. For the EHNPGP model, this facilitates determining the Nash equilibrium solutions, which could be initiated from any initial feasible points. The proposed algorithm also guarantees the system's distributed nature.

8. Numerical results support the superiority of the proposed model versus the previous ones. It has been demonstrated that our scheme not only ensures the desired SIR requirement but also allows distributing the minimised power to every CS.

\subsection{Organisation}

The remaining of this paper is outlined as follows: Section 2 demonstrates the general system model along with game formulation. Section 3 presents a mathematically ratified system for the recommended equations. The recommended algorithm is elucidated in Section 4. Section 5 shows the mathematical observations and the throughput performance through which green transmission algorithm insights are attained. Section 6 explores the drawbacks of this study and the prospects of improvement. Section 7 presents the conclusion and summary of the main findings.

\section{System Model and Problem Formulation}

This article offers a green transmission model pertaining to the next-generation $5 \mathrm{G}$ via a wireless CSN scenario. Fig. 1 presents the CSN scenario where there is one PU and $N$ cognitive users (CUs). The wireless link signal represents the SWIPT system, shown in Fig. 2 , which can be segmented into two components. The first one refers to the information while the second one refers to transferring of power since we cannot simultaneously apply radio frequency mutually [30]. The secondary users (cognitive users) possess sensor nodes that can perform spectrum sensing. We have assumed that each cognitive sensor user has the ability to harvest energy and is geared with an energy storage device. Then, denote $N=$ $\{1,2, \ldots, N\}$ defining the set of CSs. 


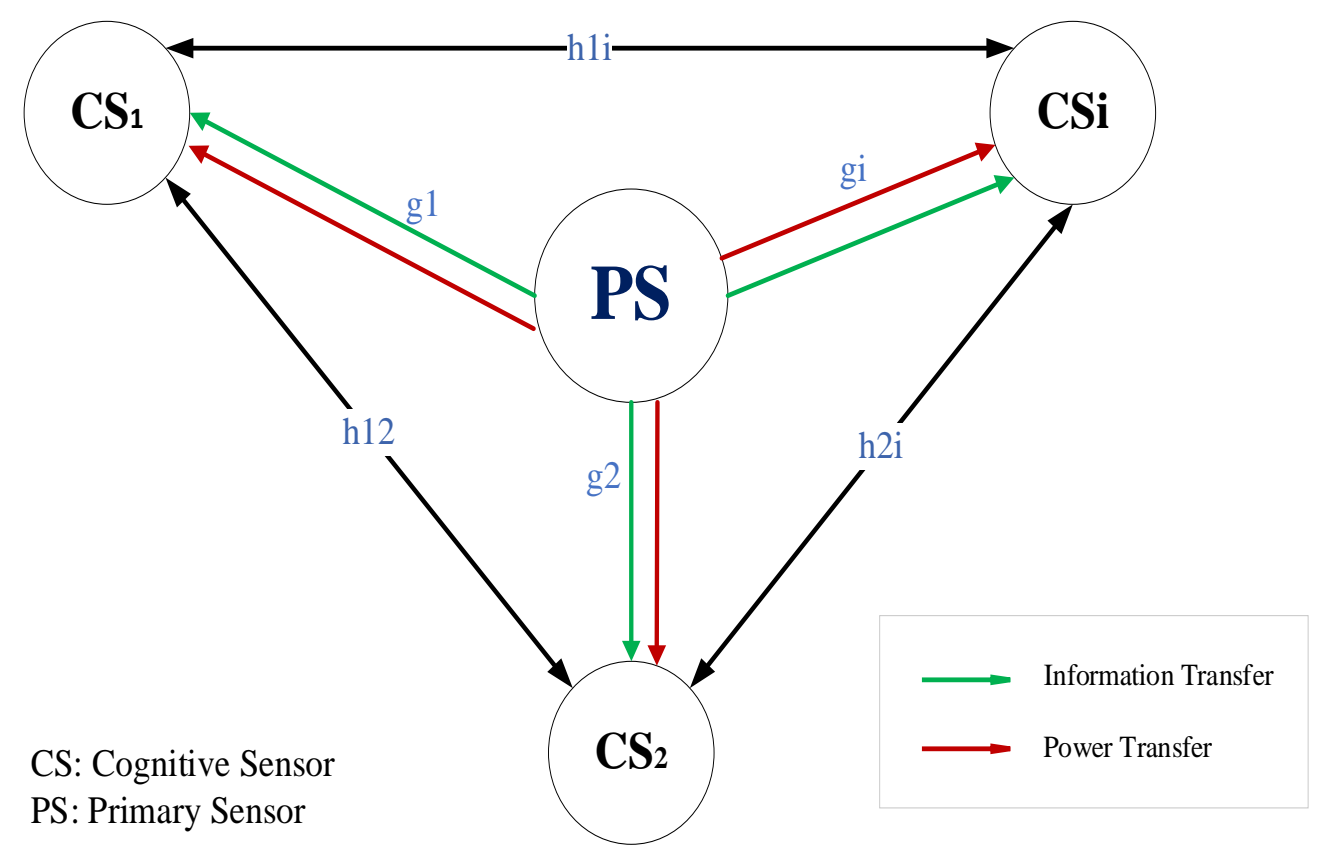

Fig. 1. System model

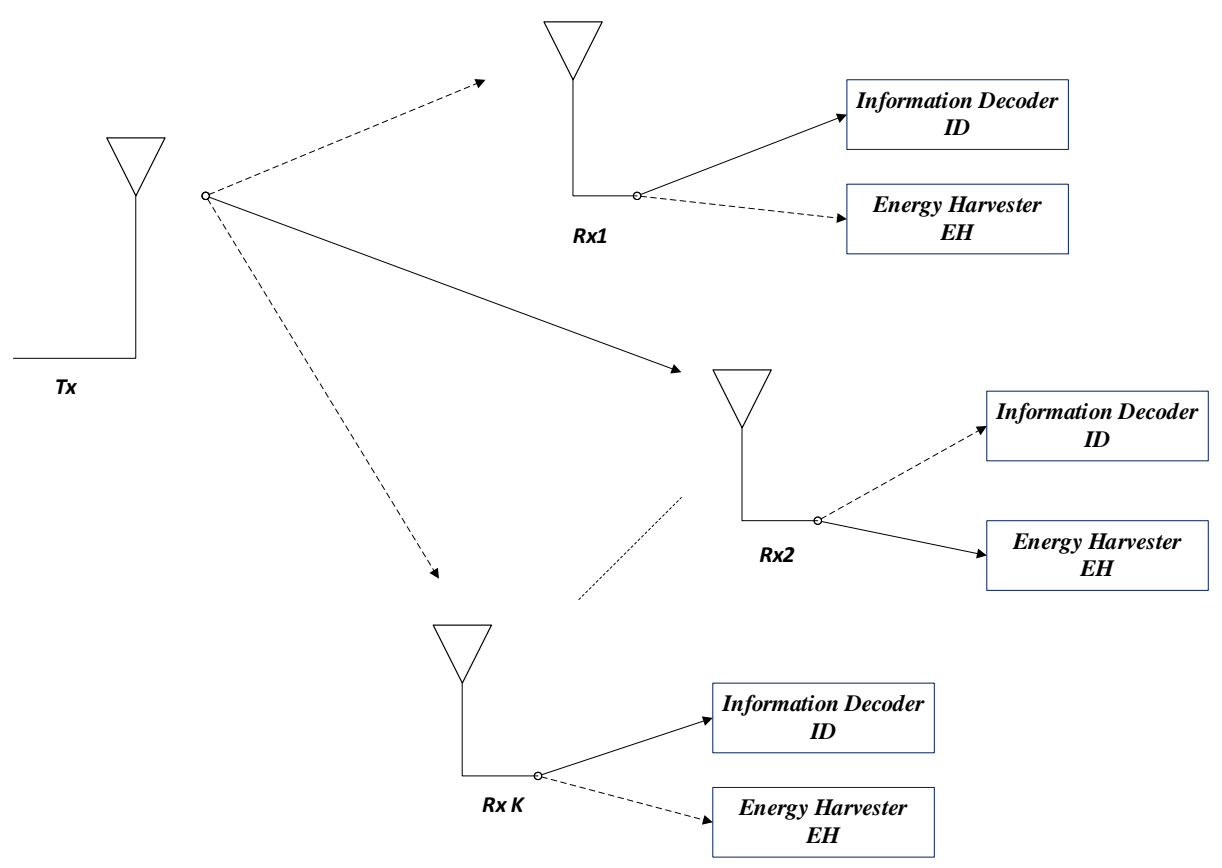

Fig. 2. SWIPT system model

The PU operates in the licensed band, while the band is shared with the secondary users [31]. Two modes are available for the PU: idle mode and data transmission mode, as presented in Fig. 3a. We take into account the total of transmission state period and its adjacent idle state duration that can be defined as an analytical interval $T$ since the PU state constantly tends to 
switch from the transmission mode to the idle mode. We also assume that the PU will not maintain a single state for long periods of time, i.e. identical to the real state.

\begin{tabular}{|l|l|}
\hline Data Transmission & Idle \\
\hline
\end{tabular}

(a) Primary Sensor

\begin{tabular}{|c|c|c|}
\hline Spectrum Sensing & Energy Harvesting & Data Transmission \\
$(1-\alpha) T$ & $\beta \alpha T$ & $(1-\beta) \alpha T$ \\
\hline
\end{tabular}

(b) Secondary Sensor

Fig. 3. Stages of ith cognitive users in $\mathrm{T}$

When PU is in the data transmission mode, it is considered as a radio frequency (RF) energy provider, while in this mode, the energy can also be harvested by CUs from the PU. However, during the time $T$, CU possesses three states: energy-harvesting phase, spectrumsensing phase and data transmission phase. Amongst these states, $\alpha$ is defined as the coefficient of spectrum-sensing time divided by $T$ into non-sensing phase and sensing phase, as presented in Fig. $\mathbf{3} \mathbf{b}$, and $\beta$ can be defined as the fraction of energy-harvesting time coefficient. As denoted, in the first period $(1-\alpha) T$, cognitive sensors in the PU state collect results in a cooperative manner. Thus, in this study, the coefficient $\alpha$ is assumed to be fixed, while the power consumption $P_{\alpha}$ is considered to be a constant value.

Moreover, in the first duration $(1-\alpha) T$, the PU spectrum usage information is sensed by the CSs, thereby allowing for the collection of sensing results in a cooperative manner. Then, when the PU assumes the transmission mode, energy can be harvested by the cognitive user from the PU, wherein it is regarded that the CS does not possess its own dedicated power supply and completely relies on the radio frequency (RF) energy harvesting, and all CSs function in the half-duplex mode. Therefore, these are not able to transmit data and harvest energy simultaneously in this duration $\alpha \beta T$. Thus, the harvested energy pertaining to CSi during the duration $\alpha \beta T$ can be represented as:

$$
E_{i}=\eta * \alpha \beta T * P_{S} * h
$$

Here, $\eta$ signifies the energy harvesting efficiency, and it is assumed that the energy $E_{i}$ will be utilised completely for spectrum sensing and the next data transmission. It is also assumed that the transmission power pertaining to $\mathrm{CSi}$ is so small that it allows for effective utilisation of the harvested energy. During the rest time $\left(1-\beta_{i}\right) \alpha T$, data will be transmitted by CSi to other CSs or PU by employing the harvested energy. 


$$
\beta_{i}=\frac{\alpha p_{i}+p_{s e s}(1-\alpha)}{\alpha p_{i}+\alpha \eta P_{s}|h|^{2}}
$$

During the sensing period $P_{\alpha}(1-\alpha) T$, the transmission power of CSi can be calculated by determining the energy consumption, which can be represented as:

$$
\begin{gathered}
p_{i}=\frac{E_{i}-p_{\text {ses }}(1-\alpha) T}{\alpha(1-\beta) T} \\
=\frac{1}{1-\beta_{i}} *\left(\eta * \beta_{i} * P_{s} * h-\frac{1-\alpha}{\alpha} * p_{\text {ses }}\right)
\end{gathered}
$$

The cognitive sensors have been deployed around the PU, while the interference generated by other cognitive sensors considerably exceeds that from the PU. Therefore, the SIR of a specific cognitive sensor is measured via the effect of transmission power $p_{i}$ and energy harvest factor $\beta_{i}$ pertaining to other CSs. Thus, the SIR pertaining to the $i$ th CS can be expressed as:

$$
\gamma_{i}\left(p_{i}\right)=\frac{p_{i} h_{i}}{\sum_{j \neq i}^{N} p_{i} h_{j}+\sigma_{i}^{2}} \geq \quad \Gamma_{i} \quad, i=1,2,3, \ldots, n
$$

Here, $h$ represents the channel gain amongst cognitive sensors, and $p_{i}$ signifies the transmitted power pertaining to cognitive sensor CSi, $\mathrm{j}$ based on Equation (3). In addition, the SIR is associated with the energy harvesting coefficient vector $\beta_{i}$ based on Equation (2). $\Gamma_{i}$ signifies the threshold SIR, while $\sigma_{i}^{2}$ denotes the Gaussian noise power.

By introducing pricing functions and dissimilar utility, various works focused on the issue of power control. In [26], the utility function is defined as:

$$
\mathrm{NPNG}: U_{i}^{c}\left(p_{i}, \boldsymbol{p}_{-i}\right)=\frac{L R}{M p_{i}}\left(1-e^{-\gamma_{i} / 2}\right)^{M}-C_{1} p_{i}
$$

Here, $C_{1}$ represents the positive pricing factor. With regards to Equation (5), the same utility function was also employed in [27]. They put forward a new pricing function in which the non-cooperative power control game (NPG) is established by employing the modified shuffled frog leaping algorithm (MSFLA), as shown in Equation (6):

$$
\mathrm{NPG}-\mathrm{MSFLA}: U_{i}^{c}\left(p_{i}, \boldsymbol{p}_{-i}\right)=\frac{L R}{M p_{i}}\left(1-e^{-\gamma_{i} / 2}\right)^{M}-C_{2} e^{p_{i}}-C_{3}\left(\gamma_{i}-\Gamma_{i}\right)
$$

Here, positive pricing factors are represented by $C_{2}$ and $C_{3}$. However, the same efficiency function, which was employed by [27] and [26], is applied to non-coherent (FSK) scheme. So, the efficiency function is represented in Equation (7):

$$
f_{1}\left(\gamma_{i}\right)=\left(1-e^{-\gamma_{i} / 2}\right)^{M}
$$


Based on the sigmoid function [28], we propose a utility function as well as a newly designed pricing function, which establishes the non-cooperative power game with pricing (NPGP) by employing the efficient swarm intelligent algorithm (ESIA) as shown in Equation (8):

$$
\mathrm{NPGP}-\mathrm{ESIA}: U_{i}^{c}\left(p_{i}, \boldsymbol{p}_{-i}\right)=\frac{L R}{M p_{i}} \frac{1+e^{-\gamma_{i}}}{1+e^{\Gamma^{\Gamma_{i}-\gamma_{i}}}}-\alpha e^{\beta\left(\left(\gamma_{i} / \Gamma_{i}\right)-1\right)} \frac{p_{i}}{p^{t h}}
$$

Here, positive pricing factors are represented by $\alpha$ and $\beta$, $p^{\text {th }}$ represents the average interference power that could be measured via the mean value of $p_{i}^{t h}: p^{t h}=\left(p_{1}^{t h}+p_{2}^{t h}+\right.$ $\left.\cdots+p_{i}^{t h}\right) / N$, while the sigmoid efficiency can be represented as:

$$
f_{2}\left(\gamma_{i}\right)=\frac{1-e^{-\gamma_{i}}}{1+e^{\Gamma_{i}-\gamma_{i}}}
$$

With regards to the fair power control game, based on a utility function proposed in [29], which in turn was based on the simplified sigmoid function as utilised in [28], we introduce a new non-linear pricing function by establishing NPGP and employing a sliding model known as R-NPGP:

$$
R-N P G P: U_{i}^{c}\left(p_{i}, \boldsymbol{p}_{-i}\right)=\frac{L R}{M p_{i}} \frac{1}{1+e^{\Gamma_{i}-\gamma_{i}}}-\mu \lambda_{i} \frac{p_{i}}{p^{t h}}
$$

Here, $\lambda_{i}$ denotes another pricing factor, and $\mu$ represents a positive pricing factor that changes with CRs with regards to their generated conditions, while the efficiency function can be represented as follows:

$$
f_{3}\left(\gamma_{i}\right)=\frac{1}{1+e^{\Gamma_{i}-\gamma_{i}}}
$$

With regards to [26], an energy-efficient game-pricing model (EF-NPGP) was put forward in [23] as shown in Equation (12):

$$
E F-N P G P: \max _{p i \in P i} U_{i}^{C}\left(p_{i}, \boldsymbol{p}_{-i}\right)=\frac{L R}{M p_{i}} \exp \left(-\left(\frac{a \Gamma_{i}}{\gamma_{i}}\right)^{b}\right)-c p_{i}^{\alpha}
$$

Here, $a$ and $b$ symbolise non-negative weighting factors, while $c$ and $\alpha$ represent the pricing factors. The expression of sigmoid efficiency function can be arrived at as a ratio of target SIR, while the required signal can be expressed as: 


$$
f_{4}\left(\gamma_{i}\right)=\exp \left(-\left(\frac{a \Gamma_{i}}{\gamma_{i}}\right)^{b}\right)
$$

With regards to a new function of sigmoidal efficiency, we propose a novel utility function as well as a power function pertaining to the transmit function of the user's power pricing. In addition, sigmoid efficiency has been introduced as a fraction along with exponential ratio power multiply tuning factor (z) with all power to allow targeting SIR to be represented as in Equation (14):

$$
f_{5}\left(\gamma_{i}\right)=\frac{1}{\left(1+\exp \left(1-z \operatorname{sir}_{i}\right)\right)^{\Gamma_{i}}}
$$

$\mathrm{z}$ is the tuning factor where its change will alter the response of the efficiency function proposed. By possessing this ability to control responses, the proposed function will be more efficient than others, as shown in the comparison given in Fig. 4. We call it an optimum function because we could use it to control efficiency response as well as to control the utility function value. However, the use of our proposed sigmoidal function will be more efficient because it is easier and more efficient to deal with one equation to control the response of the efficiency function than using more equations. With regards to Equation (16), the utility function pertaining to the $\boldsymbol{i}$ th CS can be expressed as:

$$
U_{i}=\frac{L R}{M\left(p_{i}+p s e s\right)} \frac{1}{\left(1+\exp \left(1-z \operatorname{sir}_{i}\right)\right)^{\Gamma_{i}}} \frac{\text { bit }}{\text { joule }}
$$

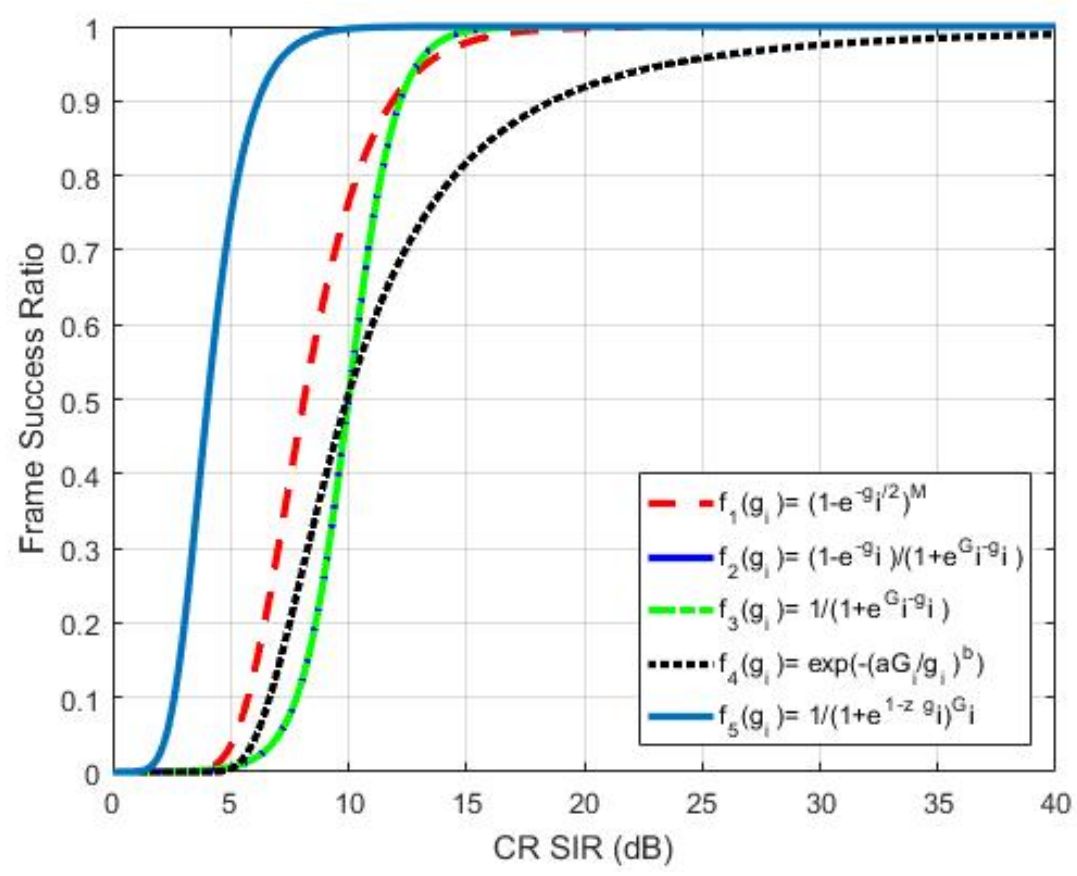

Fig. 4. Efficiency function comparison 
The utility function pertaining to Equation (15) signifies the trade-off between the throughput and battery life. It is suitable for applications where power-saving is more crucial than attaining a high throughput like green CR [32]. At cognitive radio system, we consider a fixed value of target SIR. Tuning of the proposed utility function can be done by employing the tuning factor (z). Based on the utility function maxima, optimal transmit power of the user can be varied. Fig. 4 presents how the proposed utility can be made more efficient by tuning factor versus others in the literature. The decrease in value of the parameter $\mathrm{z}$ results in an increase in utility and a decrease in the transmitting power, but it also results in a decrease in target of SIR pertaining to the system. Furthermore, the primary system can transmit the tuning factor $\mathrm{z}$ with the help of cognitive networks for adjusting the targeted SIR based on interference. When the sum of interference is about to reach the limit of interference temperature, a lower value of $\mathrm{z}$ is sent by the primary system.

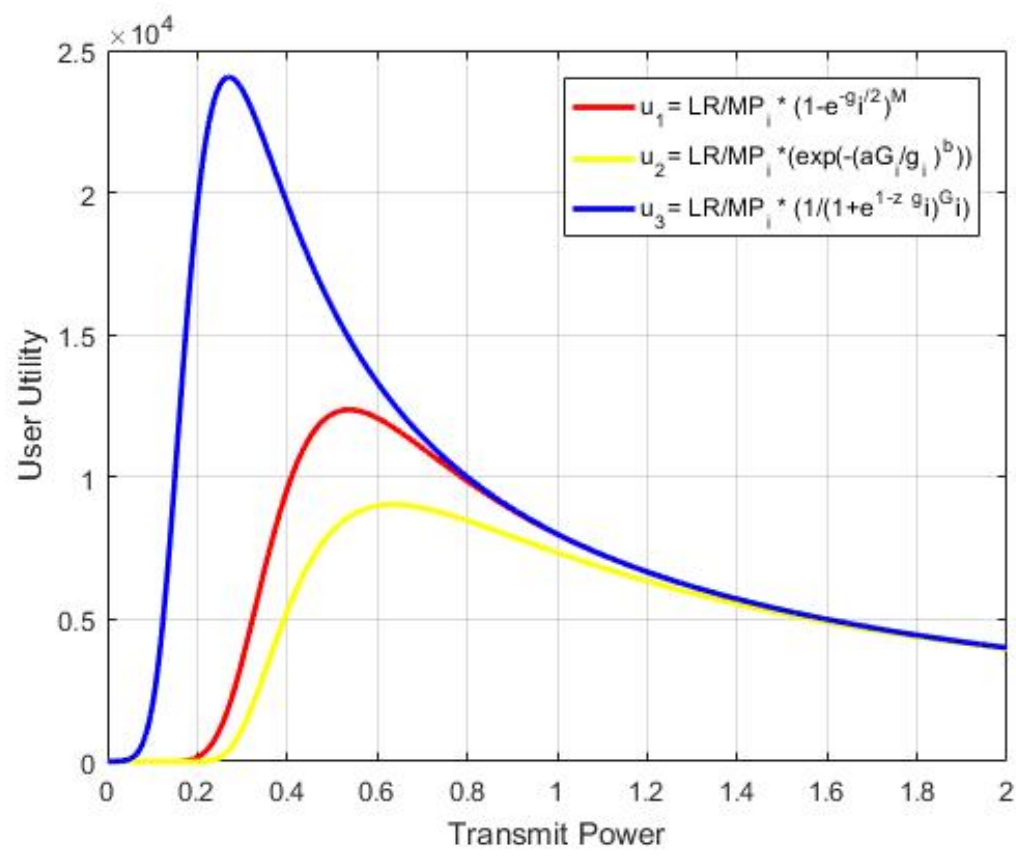

Fig. 5. Proposed utility u3 as compared to u1[33] and u2 [23]

\subsection{Game Theoretic Framework Formulation}

The game theory basically includes three key components that can be numerically denoted as $G=[M, A, U]$, where $M=\{1,2, \ldots, M\}$ signifies the finite of decision makers, $A=A_{1} \times$ $A_{2} \times \ldots \times A_{M}$ characterises the space of action that is accessible to every CSs and $U: A \rightarrow R$ denotes the utility function. Thus, the description pertaining to non-cooperative energy harvest game model could be given as follows.

Definition: The non-cooperative power energy harvest game problem pertaining to CSNs, defined as EH-NPGP, can be represented as EH - NPGP $=\left[N,\left\{P_{i}\right\},\left\{U_{i}().\right\}\right]$, where $\mathrm{N}$ represents players' set CSs, $P_{i}^{\max }$ characterises the maximum transmission power of users $i$ and $P_{i}=\left[0, P_{i}^{\max }\right]$ denotes the transmission power strategy set of users $i$. Utility function pertaining to user $i$ can be signified as $U_{i}($.$) , wherein every network user looks for ways to$ increase its own utility in a selfish way, which is selected to guarantee good QoS for CSs. 
Mathematically, user-related utility functions are represented by the number of received in terms of per joule of the energy consumption [33]:

$$
U_{i}\left(p_{i}, \boldsymbol{p}_{-i}\right)=\frac{\operatorname{LRf}\left(\gamma_{i}\right)}{M p_{i}}
$$

Here, transmitters send information to receivers in CR networks and wireless data in a frames or packets of length $M$ bits, which include $L<M$ information bits in the form of a data rate with $R$ (bits/sec), wherein $f(\gamma)$ denotes the efficiency function. Furthermore, $f(\gamma)$ needs to rely on the achieved SIR over the channel, whose value could range from 0 to 1 ) (i.e. $f(\gamma) \in[0,1])$. Moreover, power $p_{i}$ denotes the transmitted power by the user $i$.

Thus, the proposed energy harvest pertaining to the game with pricing can be represented as:

$$
\begin{aligned}
& E H-N P G P: \max _{p i \in P i} U_{i}^{C}\left(p_{i}, \boldsymbol{p}_{-i}\right)=\frac{L R}{M\left(p_{i}+p s e s\right)} \frac{1}{\left(1+\exp \left(1-z \operatorname{sir}_{i}\right)\right)^{\Gamma_{i}}} \\
&-c p_{i} \exp \left(p_{i} \alpha\right)
\end{aligned}
$$

where $\mathrm{c}$ and $\alpha$ are the pricing factors.

\subsection{Pricing Function: Setting and Formulation}

With regards to a self-organised network like CSN, users could behave in a selfish manner with the intention to maximise their own objective function, which can be achieved by increasing their own power. Pricing mechanism is a popular technique employed for managing the selfishness in CSNs. The results of Nash equilibrium due to power control of non-cooperative are deemed ineffective since they overlook the cost imposed on other terminals via the generated interfering signals. Thus, the pricing concept is introduced to motivate users regarding effective utilisation of the network resource. General expressions related to NPGP can be stated as follows:

$$
\Phi^{c}=\left[N,\left\{P_{i}\right\},\left\{U_{i}^{c}(.)\right\}\right]
$$

Here, $U_{i}^{c}($.$) represents the utility function based on pricing, which could be expressed as:$

$$
U_{i}^{c}\left(p_{i}, \boldsymbol{p}_{-i}\right)=U_{i}\left(p_{i}, \boldsymbol{p}_{-1}\right)-C_{i}\left(p_{i}, \boldsymbol{p}_{-1}\right)
$$

We subsequently employ the pricing technique for encouraging cognitive sensors to efficiently utilise network resources like power and punish users who produce higher interference to PUs or behave in a selfish manner. The pricing function could be described as follows.

Definition: The non-cooperative power game based on pricing function can be defined by EH-NPGP, which also represents the level of punishment for a player who produces certain 
amount of interference to PUs. Mathematically, the proposed pricing function can be given as:

$$
C_{i}\left(p_{i}, \boldsymbol{p}_{-i}\right)=c p_{i} \exp \left(p_{i} \alpha\right)
$$

Thus, modelling of the utility function with pricing can be done as:

$$
U_{i}^{C}\left(p_{i}, \boldsymbol{p}_{-i}\right)=\frac{L R}{M\left(p_{i}+p s e s\right)} \frac{1}{\left(1+\exp \left(1-z s i r_{i}\right)\right)^{\Gamma_{i}}}-c p_{i} \exp \left(p_{i} \alpha\right)
$$

Here, $U_{i}^{C}\left(p_{i}, \boldsymbol{p}_{-i}\right)$ defines the net utility function or the surplus function pertaining to every CSs, which could be described by the gap that exists among the payment function and its utility function.

The proposed design contributes towards applying a higher cost for the farther users because those who are farthest from the base station employ the highest power. Consequently, an exponential power function pertaining to the power transmission has been introduced in the place of the classical linear pricing. Fig. 6 demonstrates an instance defining an existing gap among the linear and the power pricing features. So, it is assumed that the power transmitted by the user differs between the maximum and minimum power strategies $[0,1]$, while numerical computation is applied to the price functions. It has been demonstrated that there is a lower power function pricing versus the linear function pricing pertaining to CSs who employ a low transmitting power for the closest users, while a high pricing cost will be associated with the CSs who employ high transmitting power for the farthest users.

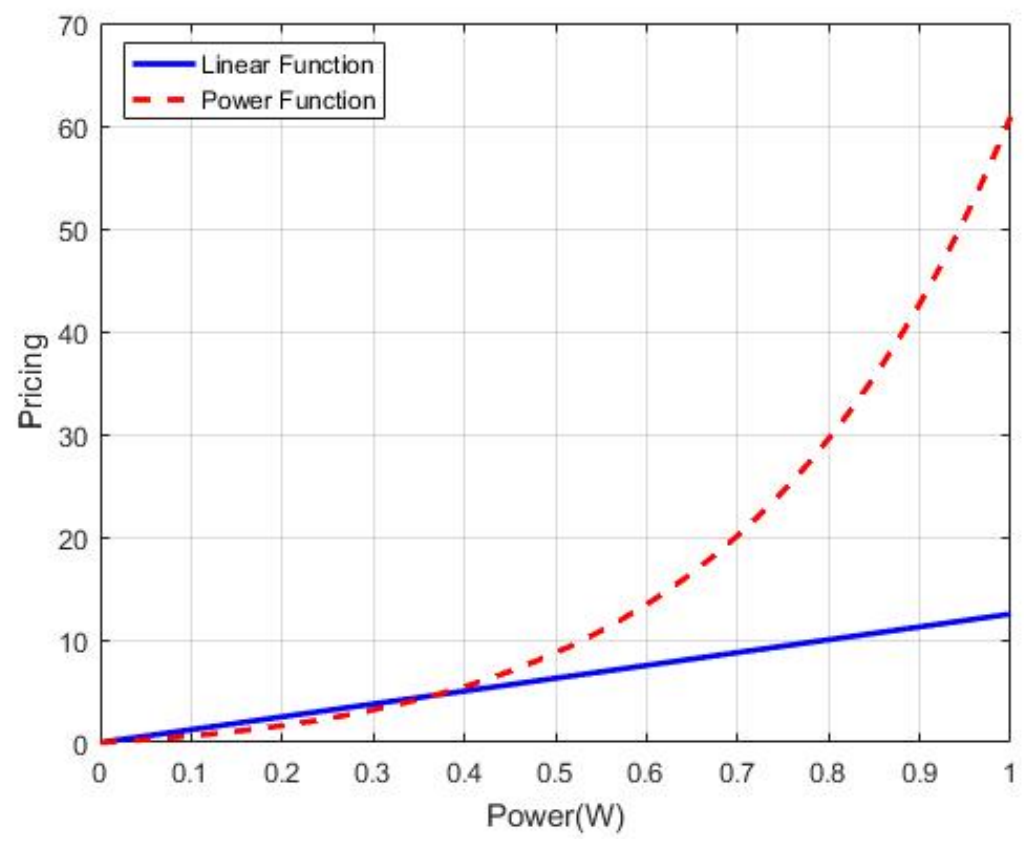

Fig. 6. Comparing linear and power function pricing 


\section{Nash Equilibrium in EH-NPGP}

This section provides a mathematical description associated with the existence as well as uniqueness pertaining to NE [34]:

Definition 3.1: In EH-NPGP, describing Nash equilibrium as a power vector, e.g. $P_{i}=$ $\left[p_{i}, \ldots, p_{i}\right]$, wherein none of the player can enhance its utility function, $U_{i}\left(p_{i}, p_{-i}\right)$; by modifying its own strategy profile unilaterally, i.e.., $p_{i}$. Numerically, the Nash equilibrium could be represented as:

$$
U_{i}\left(p_{i}, \boldsymbol{p}_{-\boldsymbol{i}}\right) \geq U_{j}\left(p_{i}, \boldsymbol{p}_{-i}\right), \quad \forall p_{i} \in \widehat{P_{l}}, \quad \forall i \in N
$$

\subsection{NE Existence}

The Nash equilibrium pertaining to EH-NPGP provides a predictable, stable result for a game which has several CSs possessing conflicting interests and then reaches a point wherein there are no requests by the CS player to modify its own strategy profile. The following theorem needs to be presented for validating the existence of the NE:

Theorem 3.1 [35] [36]: The Nash equilibrium is considered to exist in EH-NPGP = $\left[N,\left\{P_{i}\right\},\left\{U_{i}().\right\}\right]$, if the following conditions $\forall i \in N$ are satisfied:

1) The action strategy profile (i.e. $p_{i}$ ) is deemed as a nonempty, compact and convex subset.

2) The utility function $U_{i}\left(p_{i}, \boldsymbol{p}_{-i}\right)$ is deemed as a concave and continuous function over the players' strategy set.

Proof: This could be accomplished by meeting both the conditions provided in Theorem $\mathbf{3 . 1}$ in EH-NPGP by demonstrating the following evidence:

1) The first condition is readily satisfied since every CS user possesses a strategy profile defined by the minimum power as well as maximum power as expressed in Equation (15).

2) For confirming that the second condition is satisfied as well, it needs to be proved that a provided price-based utility function is concave in $p_{i}, \forall i \in N$.

Definition 3.2: According to [26], super modular definition 5, the utility function $U_{i}\left(p_{i}, \boldsymbol{p}_{-i}\right)$, characterised by the convex set $\widehat{\boldsymbol{P}_{l}}$, is concave in $P_{i}$ only in case the surplus function's second derivative is greater than $0[26,37]$.

To show this condition is true, the following set of equations $\frac{\partial^{2} u_{i}^{p}}{\partial^{2} p_{i}^{c}}>0$, must be solved $\forall i$. Thus, Lemma 3.1 needs to be satisfied.

Lemma 3.1: The price-based utility function provided in Equation (22) is deemed concave in $p_{i}, \forall i \in N$.

Assuming that the two conditions mentioned in Theorem 3.1 have been met, EH-NPGP is deemed concave in-player game, which requires one NE built into the game. 


\subsection{NE Uniqueness}

With regards to the strategy profile of the players, the proposed utility function is continuous and concave. Thus, NE was found to exist in EH-NPGP. However, at this point, a question may arise regarding the uniqueness of the existing NE. The uniqueness pertaining to the NE can be tested as follows:

Definition 3.3: The best response strategy pertains to NE's alternative definition that can be described as:

$$
B R\left(\boldsymbol{p}_{-i}\right)=\left\{p_{i}^{c} \in \hat{P}_{i}: u_{i}^{c}\left(p_{i}^{c}, \boldsymbol{p}_{-i}^{c}\right) \geq u_{i}^{c}\left(\vec{p}_{i}^{c}, \boldsymbol{p}_{-i}^{c}\right), \quad \forall \vec{p}_{i}^{c} \in \hat{P}_{i}\right\}
$$

Furthermore, the best response strategy can be defined as a set that includes just one ideal point that allows for maximising the targeted function, which is computationally defined as:

$$
p_{\left.i=\arg \max _{p_{i} \in P_{i}} U_{i}^{c}\left(p_{i}, \boldsymbol{p}_{-i}\right)\right)}
$$

Moreover, it was shown that the second derivative is greater than zero, which suggests that the maximum point can be deemed as the optimal unique point.

Theorem 3.2: The NE is unique for the non-cooperative game EH-NPGP $=\left[N,\left\{P_{i}\right\},\left\{U_{i}().\right\}\right]$.

Proof: The main feature pertaining to NE's uniqueness is aimed at showing that the best response function can be regarded as a standard function. For the proposed game EH-NPGP $=\left[N,\left\{P_{i}\right\},\left\{U_{i}().\right\}\right]$, the best response pertaining to the $i^{\text {th }}$ user is given by the power strategy pertaining to others.

For proving that NE is distinctive, a standard function should be existing as the best response function and the subsequent properties also need to be confirmed [38]:

1) For positivity: $B R\left(\boldsymbol{p}_{-i}\right)>0$.

2) For monotonicity: $\boldsymbol{p} \geq \widehat{\boldsymbol{p}}$, and then $B R\left(\boldsymbol{p}_{-i}\right) \geq B R\left(\widetilde{\boldsymbol{p}}_{-i}\right)$.

3) For scalability: $\varepsilon>1$ for all, and then $\varepsilon B R\left(\boldsymbol{p}_{-i}\right)>B R\left(\varepsilon \boldsymbol{p}_{-i}\right)$.

Through the power strategy space provided in Equation (15) and by Theorem 3.1, the first two characteristics can be verified with ease. Thus, the scalability characteristic was found to be satisfactory.

In conclusion, the best response strategy function was found to be a standard. As a result, a non-cooperative power control model was proposed to include just one unique Nash equilibrium solution, which also proves the distinctiveness of the Nash equilibrium. 


\section{EH-NPGP Algorithm}

This section presents an iterative based best response algorithm scheme that controls all transmission powers and guarantees the required SIR among all CSNs. Furthermore; it ensures Nash equilibrium opportunistically with available SIR information.

However, we suppose that each CSN updates its transmit power at time instances $t_{i}=$ $\left\{t_{i 1}, t_{i 2}, \ldots\right\}$, where $t_{i k}<t_{i(k+1)}$, and assume that the strategy set of power of the $i$ th CSN is $P_{i}=\left[P_{i}^{\min }, P_{i}^{\max }\right]$. We set an infinity small quantity $\varepsilon$ where $(\varepsilon>0)$, and by considering the proposed algorithm as given, Equation (17) generates power sequence as follows:

\section{EH-NPGP}

I. Initialise vector of transmit power $p=\left[p_{1}^{0}, p_{2}^{0}, p_{3}^{0}, \ldots, p_{N}^{0}\right]$ randomly at time $t_{0}$, along with other parameters including: $\sigma^{2}, P_{i}^{\max }, \Gamma_{i}$, pricing factors, $c$ and $\alpha$ and tuning factor, $z$.

II. Iteration step:

For all $i \in N$ when time instant $t_{k}$;

a) Update $\beta_{i}\left(t_{k}\right)$ by Equation (2)

b) Update $p_{i}\left(t_{k}\right)$ by Equation (3)

c) Update $\gamma_{i}\left(t_{k}\right)$ by Equation (4)

d) Given $p_{i}\left(t_{k-1}\right)$, consider the best response of power strategy $r_{i}\left(t_{k}\right)$ from BR algorithm based on $r_{i}\left(t_{k}\right)=\arg \max _{p_{i} \in P_{i}} u_{i}^{C}\left(p_{i}, \boldsymbol{p}_{-i}\left(t_{k-1}\right)\right)$

e) Assign the transmit power as $p_{i}\left(t_{k}\right)=\min \left(r_{i}\left(t_{k}\right), p_{i}^{\max }\right)$

III. Convergence step:

If $\left\|p\left(t_{k}\right)-p\left(t_{k-1}\right)\right\| \leq \varepsilon$, declare Nash equilibrium and stop iteration as $p\left(t_{k}\right)$; otherwise: $k=k+1 \&$ go to step II

IV. End

$r_{i}\left(t_{k}\right)$ is used here as the representative of the collection of the best transmit powers that correspond to the $i$ th CSN. This can be obtained when the objective function is applied with $\mathrm{BR}$ algorithm during time instant $k$. This is performed as a response to the interference vector $p_{-i}\left(t_{k-1}\right)$. One should remember that the $i$ th CSN is responsible for the optimisation of the net utility given the power strategy space for the EH-NPGP. The algorithm can be used to determine the $i$ th CSN's transmit power by choosing the lower values of power from all the possibilities set by the algorithm. The algorithm can then be used to separately solve the maximum for every CSN's objective.

Moreover, the proposed algorithm is based on power allocation using pricing function. Hence, the computational complexity directly depends on the number of users and the available channels which resulted in $O(\log (N))$. Fig. 7 illustrates the flowchart of the proposed algorithm: 


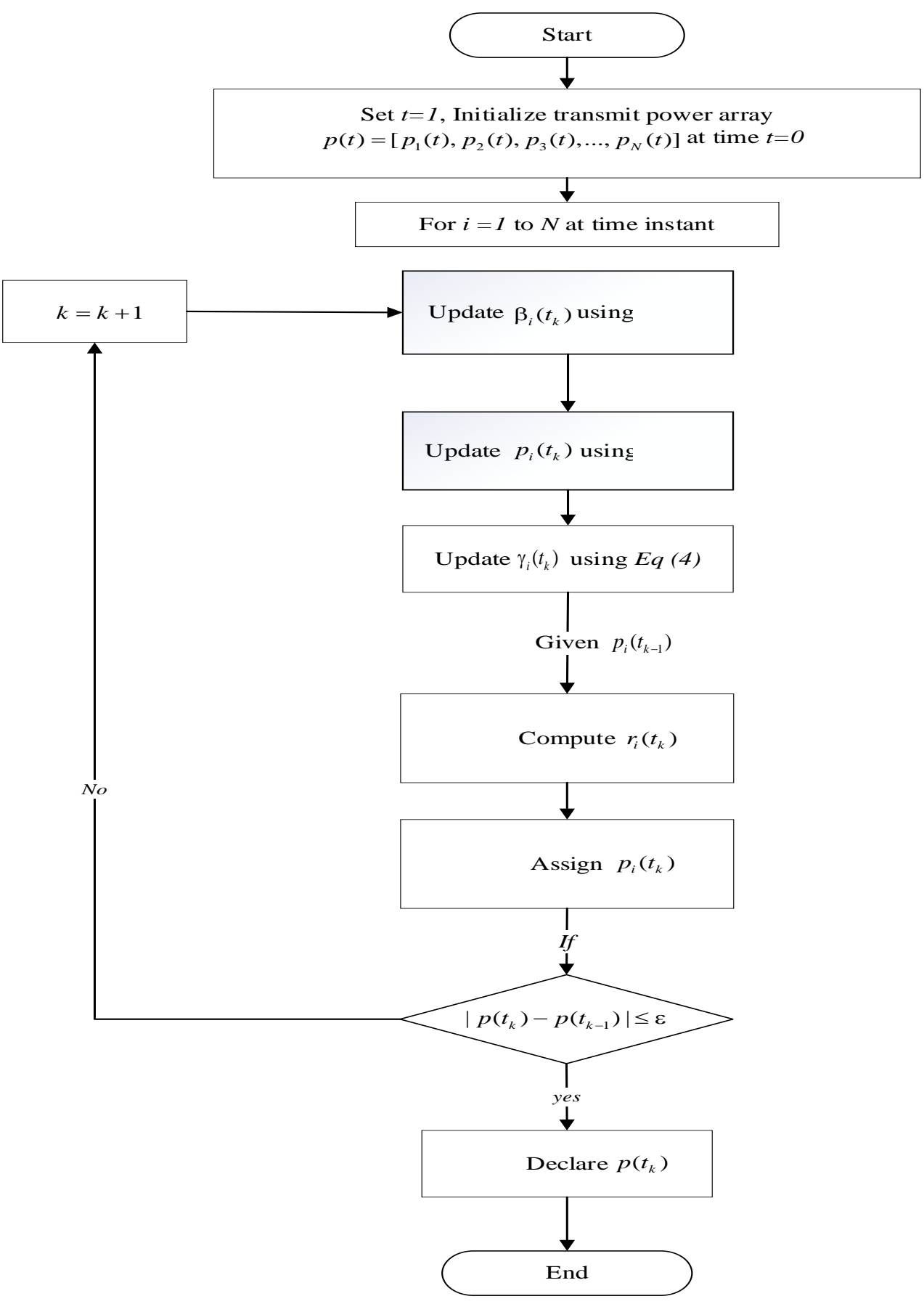

Fig. 7. Proposed algorithm illustration 


\section{Results and Discussion}

CSN scenario and simulation settings are defined in this section. The NE convergence for both SIR and power is also verified. There is a similarity of the suggested power algorithm with the algorithm given in NPG-MSFLIA [27], R-NPGP [29], NPG-ESIA [28], and EHNPGP [23] as a way of showing the superiority of the suggested power algorithm.

The utility functions employed for the comparisons are presented in Equations (6), (8), (10) and (12). For attaining the utility functions' Nash equilibrium solution as a way to introduce the benefits of the proposed utility function, the same numerical computation was implemented. Table 1 lists the constant system parameters utilised in the simulation.

Table 1. System Parameters

\begin{tabular}{|c|c|}
\hline Parameter & Value \\
\hline Players number & 7 \\
\hline $\mathrm{M}$ & 80 \\
\hline $\mathrm{L}$ & 64 \\
\hline $\mathrm{G}$ & 70 \\
\hline $\mathrm{R}$ & $10 \mathrm{kbps}$ \\
\hline$\sigma^{2}$ & $4 \mathrm{e}-8 \mathrm{Watts}$ \\
\hline Maximum power constraint, $P_{i}^{\text {max }}$ & 1 Watts \\
\hline$P_{s}$ & $20 \mathrm{e}-3$ Watt \\
\hline Pses & $10 \mathrm{e}-2$ Watt \\
\hline Efficiency $\eta$ & 0.5 \\
\hline Target SIR, $\Gamma_{i}$ & 10 \\
\hline Pricing factors, $c \& \alpha$ & $1 \mathrm{e} 4,2.5$ \\
\hline Tuning factor, $z$ & 0.9 \\
\hline
\end{tabular}

There are no more than seven CSs in the structure, and the distance from the base station is presented as $d=[368,490,580,630,720,810,950] \mathrm{m}$.

A simple propagation model utilised in this study that uses path gains as deterministic functions, $\beta$, represents path loss exponent. The following formula can then be used to compute the cognitive base station (CBS) based on the distance between the cognitive users $i$ :

$$
h_{i}=\frac{K}{d_{i}^{\beta}}
$$

In this formula, $d_{i}$ represents the gap between the base station and the $i$ th user, and $\beta$ represents the path loss exponent that normally has a value of 2, but can range between 2 and 6. $K$ is a constant equal to 0.097 ; this value is chosen for a transmit power of $1 \mathrm{~W}$ when a CSN works at $950 \mathrm{~m}$ from CBS in a system that has seven CSs which all work with $\gamma^{*}$. For the simulation of this study, the sensor users begin with an initial power $p_{i}^{(0)}=2.22 \times$ $11 w$ for all algorithms and $\varepsilon=10^{-5}$. 


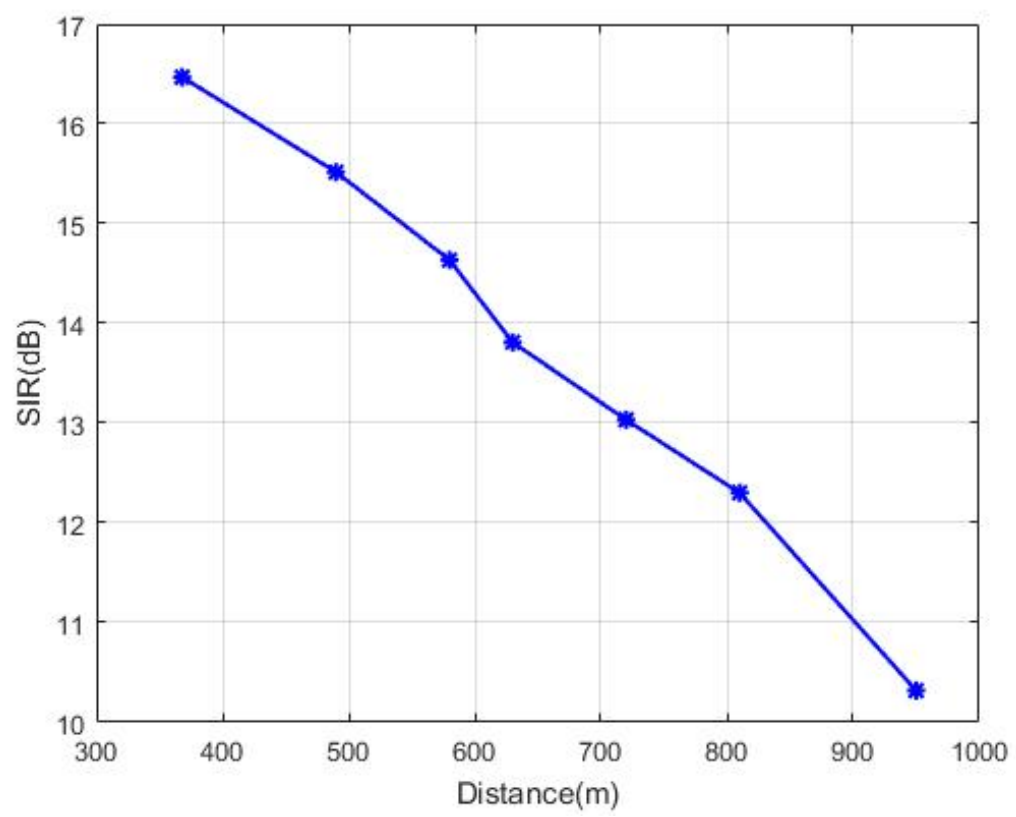

Fig. 8. SIR vs. distance for each user

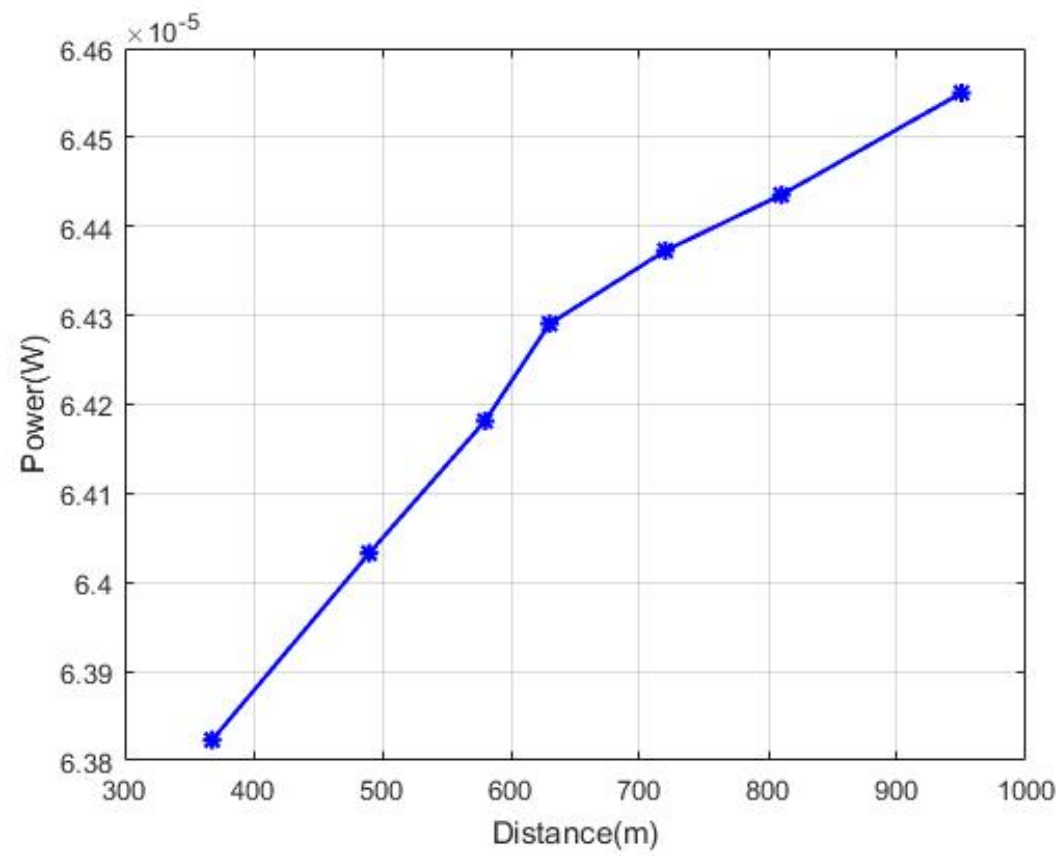

Fig. 9. Power vs. distance for each user

Fig. 8 illustrates the findings of SIR at the Nash equilibrium. These results were obtained by CS based on the gap among each base station and CS. All CS users keep the value of their SIR higher than the target value $\left(\Gamma_{i}=10\right)$. One can decrease the SIR value by lengthening the gap for all algorithms. Among the algorithms, the differences values of SIR revealed that 
the proposed algorithm EH-NPGP has more efficiency than other algorithms when the SIR values are at their highest. The outermost CSs have the highest power consumption and they are representative of the origin of interference. The proposed algorithm is designed and implemented to charge the farthest sensors at the highest cost. Fig. 9 demonstrates the curves of power transmitted in Watts with the distance between base station and the CS for all algorithms. In such cases, power transmission experiences a gradual increase as the distance of the user increases. One may observe that the suggested EH-NPGP has the lowest power transmission curve compared to the other algorithms in literature such as NPG-MSFLIA, RNPGP, NPGP-ESIA and EF-NPGP.

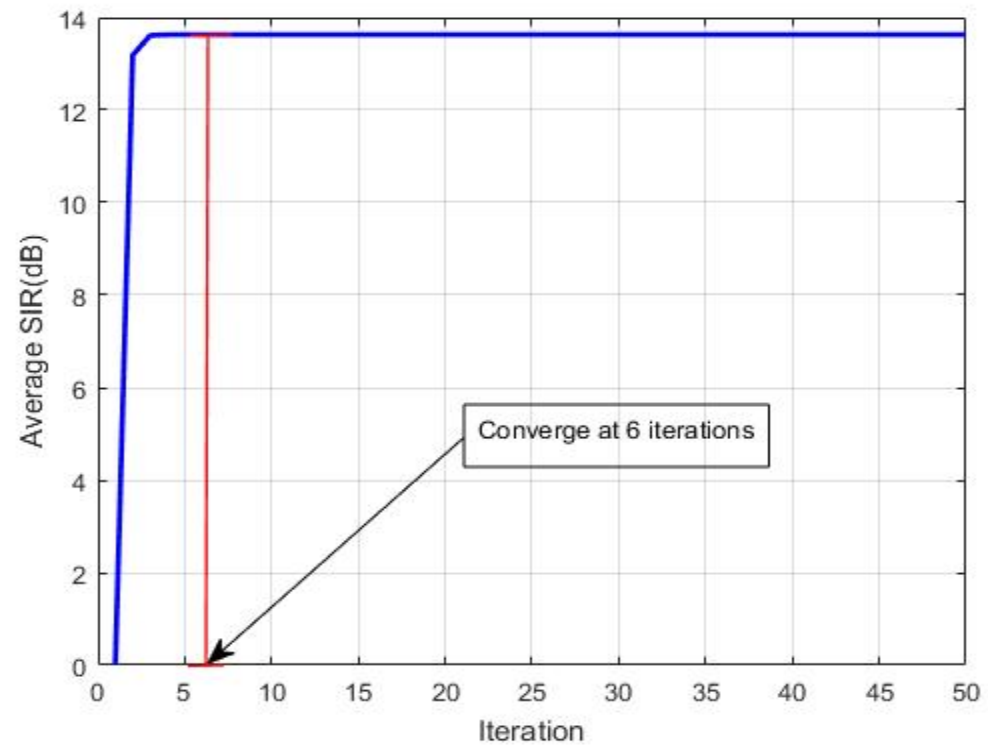

Fig. 10. Proposed Algorithm Convergence of Average SIR

The SIR of CSN users of the network algorithm simulation is shown in Table 2. Furthermore, it is demonstrated in the table that the EH-NPGP, with the proposed price function, achieves higher SIR value than the other algorithms. It is seen that SIR is smaller in the last two cognitive sensors because of the applied higher cost. So, it can achieve a better equilibrium.

Table 2. CSN Final SIR

\begin{tabular}{|c|c|c|c|c|c|}
\hline Users & $\begin{array}{c}\text { Final SIR of } \\
\text { NPG-MSFLIA } \\
{[27]}\end{array}$ & $\begin{array}{c}\text { Final SIR of } \\
\text { NPG-ESIA } \\
\text { [28] }\end{array}$ & $\begin{array}{c}\text { Final SIR of R- } \\
\text { NPGP [29] }\end{array}$ & $\begin{array}{c}\text { Final SIR of } \\
\text { EF-NPGP [23] }\end{array}$ & $\begin{array}{c}\text { Final SIR of } \\
\text { Proposed EH- } \\
\text { NPGP }\end{array}$ \\
\hline 1 & 12.41 & 12.42 & 12.42 & 12.69 & 16.47 \\
\hline 2 & 12.4 & 12.43 & 12.43 & 12.69 & 15.51 \\
\hline 3 & 12.4 & 12.43 & 12.43 & 12.69 & 14.63 \\
\hline
\end{tabular}




\begin{tabular}{|c|c|c|c|c|c|}
\hline 4 & 12.39 & 12.43 & 12.43 & 12.69 & 13.80 \\
\hline 5 & 12.37 & 12.42 & 12.42 & 12.68 & 13.03 \\
\hline 6 & 12.33 & 12.4 & 12.4 & 12.66 & 12.30 \\
\hline 7 & 12.26 & 12.3 & 12.3 & 12.4 & 10.31 \\
\hline
\end{tabular}

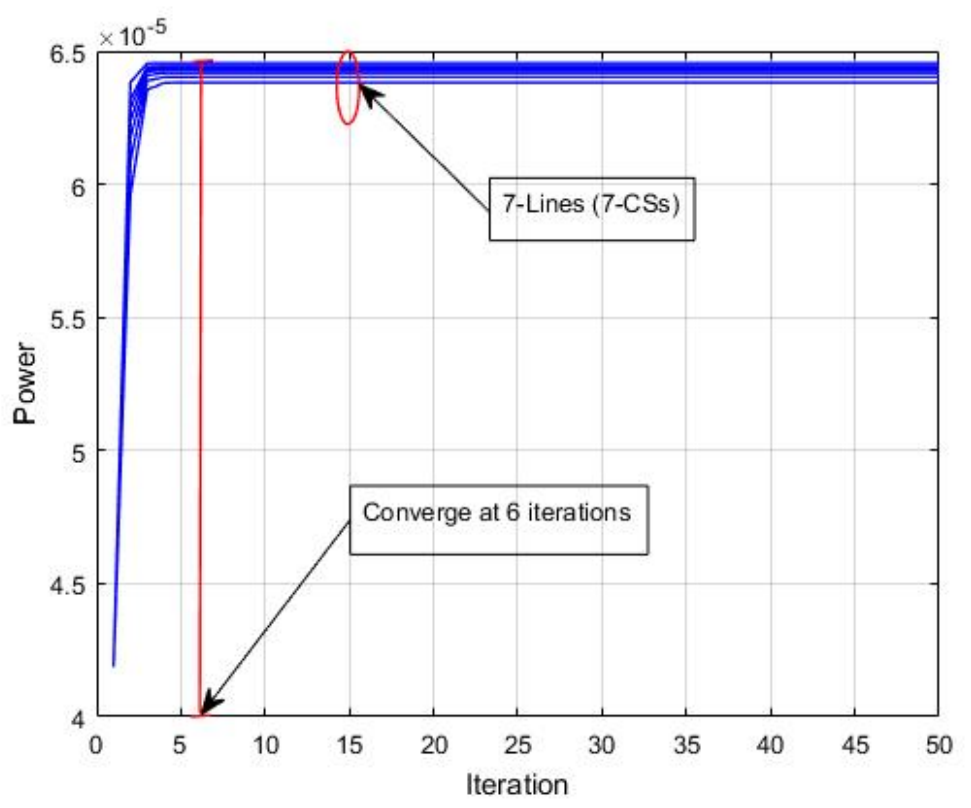

Fig. 11. Convergence of the proposed power algorithm

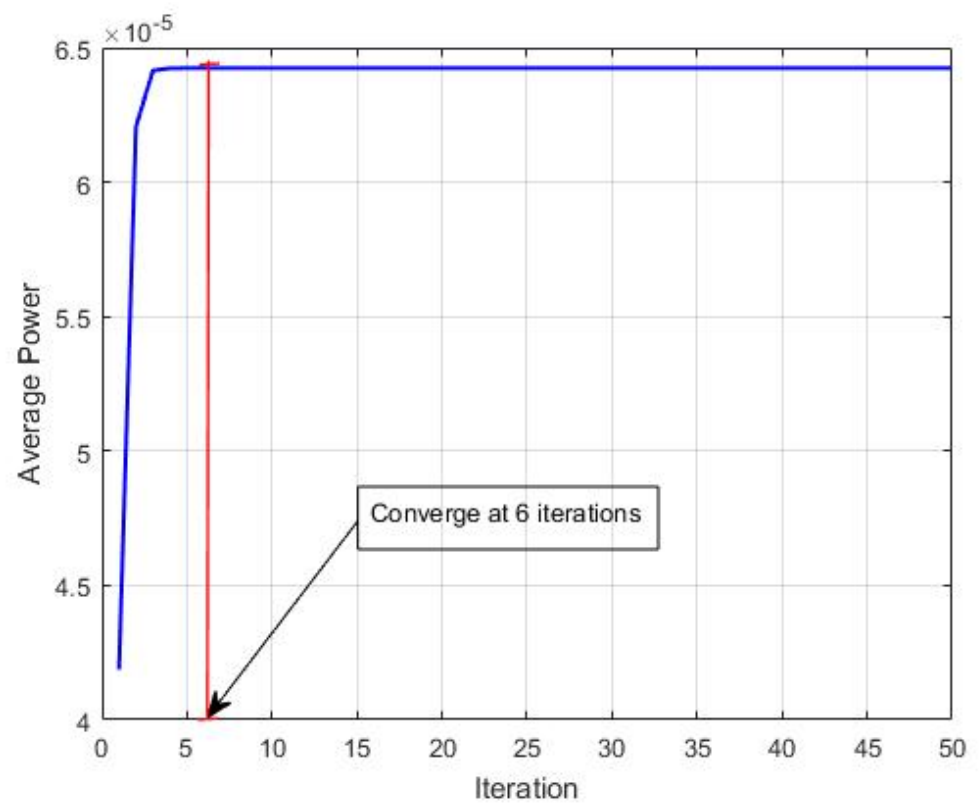

Fig. 12. Convergence of average power in the proposed algorithm 
Fig. 11 demonstrates the proposed algorithm convergence, which proves that the allocated power in a network of seven cognitive sensors performs better than the others because it has faster convergence. It only needs six iterations to converge, compared with 333, 323, 360 and 133 for NPG-MSFLA, R-NPGP, NPGP-ESIA and EF-NPGP, respectively. Moreover, the average SIR and average power were tested and compared with the method of algorithms comparison to determine the reduction of average power and the convergence speed of algorithms. In this test, the iteration time needed to obtain the Nash equilibrium is represented by the horizontal axis, while the average power and average SIR are represented by the vertical axis. Table 2 demonstrates that all algorithms were able to achieve approximately the same average SIR value without any considerable differences. However, they have unequal speeds of convergence. The algorithm proposed in this study obtained the Nash equilibrium after six iterations. This is illustrated in Fig. 10. On the other hand, NPGMSFLA, NPGP-ESIA, R-NPGP and EF-NPGP required 333, 360, 323 and 133 iterations, respectively, to achieve this. Fig. 12 demonstrates the average transmit power curve obtained by the suggested algorithm. Fig. 12 also shows that the suggested EH-NPGP algorithm has significantly reduced average power consumption than other algorithms. This is necessary for building promising $5 \mathrm{G}$ wireless networks. The study managed to lower the transmitted power successfully from Watt to micro-Watt, as illustrated in Fig. 12. The outcomes presented in Fig. 12 are indicators of the amount of interference observed at the primary system based on the proposed EH-NPGP, given that it is the lowest among all the compared algorithms in the literature. Thus, the proposed EH-NPGP algorithm characteristics make it the best option for the maximisation of QoS guarantees and spectrum sharing in both systems. Fig. 12 demonstrates clearly the speed convergence of the suggested algorithm, which indicates that the suggested EH-NPGP is faster than the other algorithms, as observed in Table 3. This can also be attributed to the values of average power in Watt and the numbers of repetitions for all algorithms.

Table 3. Comparison of average power and NE convergence

\begin{tabular}{|c|c|c|}
\hline Algorithm & Average Power (W) & Iterations \\
\hline NPG-MSFLA [27] & 0.2321 & 333 \\
\hline NPGP-ESIA [28] & 0.2319 & 360 \\
\hline R-NPGP [29] & 0.2287 & 323 \\
\hline EF-NPGP [23] & 0.1926 & 133 \\
\hline EH-NPGP & $6.4241 \mathrm{e}-05$ & 6 \\
\hline
\end{tabular}

\section{Research Limitation}

When there is no pricing, there is a maximisation of all the above utilities for the same value of SIR. The SIR value is determined based on the energy harvest factor and efficiency function designs, which depend on the characteristics of the system. When the pricing function is implemented and the value of pricing factor rises, cognitive sensors are able to achieve lower SIR, higher utilities and lower power. As a result of the pricing, it was observed that for all CSNs, SIR is no longer equal at the equilibrium. It was also observed that a user achieved higher SIR when it was closer to the base station. The lowering of CSNs' SIR, the rise of CSN utilities and the increased algorithm speed of convergence depend on the chosen values of pricing factors $c$ and $\alpha$. 


\section{Conclusion}

This study determined the best way to use the energy harvest time for maximising the radio frequency energy efficiency. We proposed a novel EH-NPGP algorithm that is built on noncooperative game pricing theory. The proposed algorithm has a convergence that has been verified by simulation. We also derived mathematical proof for the presence and distinctiveness of the NE. One of the most apparent benefits of the suggested energy harvesting algorithm is its ability to more quickly converge to the NE compared to those in the previous works. The proposed algorithm is therefore more appropriate for a functional distribution application. Furthermore, pricing is a vital component of CSNs for avoiding cheating behaviour among players. Thus, one can achieve better performance for both primary and secondary CSN users. Going forward, we recommend that the researchers consider the proposed algorithm and mathematical model for other scenarios and techniques to get a more enhanced system and harvest more energy for achieving the green scenario for wireless networks in the nearest-future $5 \mathrm{G}$ and beyond.

\section{References}

[1] S. Zhang, X. Xu, Y. Wu, and L. Lu, "5G: Towards energy-efficient, low-latency and highreliable communications networks," in Proc. of 2014 IEEE International Conference on Communication Systems, pp. 197-201, 2014. Article (CrossRef Link)

[2] E. Baccarelli, P. G. V. Naranjo, M. Scarpiniti, M. Shojafar, and J. H. Abawajy, "Fog of everything: Energy-efficient networked computing architectures, research challenges, and a case study," IEEE access, vol. 5, pp. 9882-9910, 2017.

[3] F. Mukhlif, K. A. B. Noordin, A. M. Mansoor, and Z. M. Kasirun, "Green transmission for C-RAN based on SWIPT in 5G: a review," Wireless Networks, vol. 25, pp. 2621-2649, 2019. Article (CrossRef Link)

[4] A. Osseiran, F. Boccardi, V. Braun, K. Kusume, P. Marsch, M. Maternia, et al., "Scenarios for 5G Mobile and Wireless Communications: The Vision of the METIS Project," Ieee Communications Magazine, vol. 52, pp. 26-35, May 2014. Article (CrossRef Link)

[5] Z. Huang, Q. Niu, S. Xiao, and T. Li, "Energy harvesting algorithm considering max flow problem in wireless sensor networks," Computer Communications, vol. 150, pp. 626-633, 2020/01/15/ 2020. Article (CrossRef Link)

[6] D. Y. Lin and Q. Wang, "A game theory based energy efficient clustering routing protocol for WSNs," Wireless Networks, vol. 23, pp. 1101-1111, May 2017. Article (CrossRef Link)

[7] S. Luitel and S. Moh, "Energy-Efficient Medium Access Control Protocols for Cognitive Radio Sensor Networks: A Comparative Survey," Sensors, vol. 18, p. 3781, Nov 2018. Article (CrossRef Link)

[8] S. Sheikhzadeh, M. R. Javan, and N. Mokari, "Cooperative multiple access cognitive radio transmission with renewable energy sources," Physical Communication, vol. 40, p. 101049, 2020. Article (CrossRef Link)

[9] J. Mitola and G. Q. Maguire, "Cognitive radio: Making software radios more personal," Ieee Personal Communications, vol. 6, pp. 13-18, Aug 1999. Article (CrossRef Link)

[10] Y. Arjoune and N. Kaabouch, "A Comprehensive Survey on Spectrum Sensing in Cognitive Radio Networks: Recent Advances, New Challenges, and Future Research Directions," Sensors, vol. 19, p. 126, Jan 2019. Article (CrossRef Link)

[11] M. S. Adam, M. H. Anisi, and I. Ali, "Object tracking sensor networks in smart cities: Taxonomy, architecture, applications, research challenges and future directions," Future Generation Computer Systems, vol. 107, pp. 909-923, 2020. Article (CrossRef Link) 
[12] R. Khan, I. Ali, M. Zakarya, M. Ahmad, M. Imran, and M. Shoaib, "Technology-assisted decision support system for efficient water utilization: a real-time testbed for irrigation using wireless sensor networks," IEEE Access, vol. 6, pp. 25686-25697, 2018.

Article (CrossRef Link)

[13] X. Lu, P. Wang, D. Niyato, D. I. Kim, and Z. Han, "Wireless Networks With RF Energy Harvesting: A Contemporary Survey," IEEE Communications Surveys \& Tutorials, vol. 17, pp. 757-789, 2015. Article (CrossRef Link)

[14] F. Mukhlif, K. A. B. Nooridin, Y. A. A.-. Gumaei, and A. S. A.-. Rassas, "Energy Harvesting For Efficient 5G Networks," in Proc. of 2018 International Conference on Smart Computing and Electronic Enterprise (ICSCEE), pp. 1-5, 2018. Article (CrossRef Link)

[15] A. A. Babayo, M. H. Anisi, and I. Ali, "A review on energy management schemes in energy harvesting wireless sensor networks," Renewable and Sustainable Energy Reviews, vol. 76, pp. 1176-1184, 2017. Article (CrossRef Link)

[16] I. Ali, A. Gani, I. Ahmedy, I. Yaqoob, S. Khan, and M. H. Anisi, "Data collection in smart communities using sensor cloud: recent advances, taxonomy, and future research directions," IEEE Communications Magazine, vol. 56, pp. 192-197, 2018. Article (CrossRef Link)

[17] L. Liu, R. Zhang, and K.-C. Chua, "Wireless information transfer with opportunistic energy harvesting," IEEE Transactions on Wireless Communications, vol. 12, pp. 288-300, 2013. Article (CrossRef Link)

[18] B. Gurakan, O. Ozel, J. Yang, and S. Ulukus, "Energy Cooperation in Energy Harvesting Communications," Ieee Transactions on Communications, vol. 61, pp. 4884-4898, Dec 2013. Article (CrossRef Link)

[19] Y. K. Chia, S. M. Sun, and R. Zhang, "Energy Cooperation in Cellular Networks with Renewable Powered Base Stations," Ieee Transactions on Wireless Communications, vol. 13, pp. 6996-7010, Dec 2014. Article (CrossRef Link)

[20] K. B. Huang and E. Larsson, "Simultaneous Information and Power Transfer for Broadband Wireless Systems," Ieee Transactions on Signal Processing, vol. 61, pp. 5972-5986, Dec 2013. Article (CrossRef Link)

[21] D. W. K. Ng, E. S. Lo, and R. Schober, "Wireless Information and Power Transfer: Energy Efficiency Optimization in OFDMA Systems," Ieee Transactions on Wireless Communications, vol. 12, pp. 6352-6370, Dec 2013. Article (CrossRef Link)

[22] E. Hossain, D. Niyato, and Z. Han, Dynamic Spectrum Access and Management in Cognitive Radio Networks, Cambridge University Press, 2009. Article (CrossRef Link)

[23] Y. A. Al-Gumaei, K. A. Noordin, A. W. Reza, and K. Dimyati, "A Novel Utility Function for Energy-Efficient Power Control Game in Cognitive Radio Networks," Plos One, vol. 10, Aug 2015. Article (CrossRef Link)

[24] Z. Zhijin, P. Zhen, Z. Shilian, and S. Junna, "Cognitive radio spectrum allocation using evolutionary algorithms," Wireless Communications, IEEE Transactions on, vol. 8, pp. 44214425, 2009. Article (CrossRef Link)

[25] Y. Wang, Q. Zhang, Y. Zhang, and P. Chen, "Adaptive resource allocation for cognitive radio networks with multiple primary networks," EURASIP Journal on Wireless Communications and Networking, vol. 2012, p. 252, 2012. Article (CrossRef Link)

[26] C. U. Saraydar, N. B. Mandayam, and D. J. Goodman, "Efficient power control via pricing in wireless data networks," Ieee Transactions on Communications, vol. 50, pp. 291-303, Feb 2002. Article (CrossRef Link)

[27] X. D. Zhang, Y. F. Zhang, Y. H. Shi, L. Zhao, and C. R. Zou, "Power control algorithm in cognitive radio system based on modified Shuffled Frog Leaping Algorithm," AeuInternational Journal of Electronics and Communications, vol. 66, pp. 448-454, 2012. Article (CrossRef Link)

[28] Y. H. Kuo, J. H. Yang, and J. Chen, "Efficient swarm intelligent algorithm for power control game in cognitive radio networks," Iet Communications, vol. 7, pp. 1089-1098, Jul 2013. Article (CrossRef Link) 
[29] X. Z. Xie, H. L. Yang, A. V. Vasilakos, and L. He, "Fair Power Control Using Game Theory with Pricing Scheme in Cognitive Radio Networks," Journal of Communications and Networks, vol. 16, pp. 183-192, Apr 2014. Article (CrossRef Link)

[30] K. Lee and J. P. Hong, "Energy-Efficient Resource Allocation for Simultaneous Information and Energy Transfer With Imperfect Channel Estimation," IEEE Transactions on Vehicular Technology, vol. 65, pp. 2775-2780, 2016. Article (CrossRef Link)

[31] M. Y. Zhao, Y. F. Wei, Q. Li, M. Song, and N. N. Liu, "Energy Harvesting Time Coefficient Analyze for Cognitive Radio Sensor Network Using Game Theory," Human Centered Computing, Hcc 2017. vol. 10745, pp. 318-329, 2018. Article (CrossRef Link)

[32] F. Meshkati, M. Chiang, H. V. Poor, and S. C. Schwartz, "A game-theoretic approach to energy-efficient power control in multicarrier CDMA systems," Ieee Journal on Selected Areas in Communications, vol. 24, pp. 1115-1129, Jun 2006. Article (CrossRef Link)

[33] D. Goodman and N. Mandayam, "Power control for wireless data," Ieee Personal Communications, vol. 7, pp. 48-54, Apr 2000. Article (CrossRef Link)

[34] J. O. Neel, J. H. Reed, and R. P. Gilles, "Convergence of cognitive radio networks," in Proc. of 2004 IEEE Wireless Communications and Networking Conference (IEEE Cat. No. 04TH8733), pp. 2250-2255, 2004. Article (CrossRef Link)

[35] B. B. Wang, Y. L. Wu, and K. J. R. Liu, "Game theory for cognitive radio networks: An overview," Computer Networks, vol. 54, pp. 2537-2561, Oct 2010. Article (CrossRef Link)

[36] O. B. Abdul-Ghafoor, M. Ismail, R. Nordin, and A. Abd El-Saleh, "Resource Allocation in Spectrum Sharing ad-hoc Cognitive Radio Networks Based on Game Theory: An Overview," Ksii Transactions on Internet and Information Systems, vol. 7, pp. 2957-2986, Dec 2013. Article (CrossRef Link)

[37] J. S. Pang, G. Scutari, D. P. Palomar, and F. Facchinei, "Design of Cognitive Radio Systems Under Temperature-Interference Constraints: A Variational Inequality Approach," Ieee Transactions on Signal Processing, vol. 58, pp. 3251-3271, Jun 2010. Article (CrossRef Link)

[38] R. D. Yates, "A FRAMEWORK FOR UPLINK POWER-CONTROL IN CELLULAR RADIO SYSTEMS," Ieee Journal on Selected Areas in Communications, vol. 13, pp. 13411347, Sep 1995. Article (CrossRef Link) 


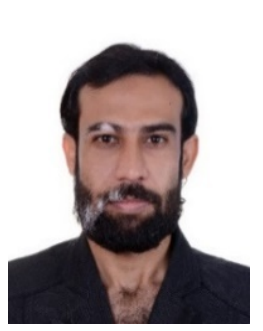

FADHIL MUKHLIF completed my BSc. in Electrical Engineering from University of Tikrit, Iraq in 2010 and MSc. in Telecommunication Engineering from University Technical Malaysia Melaka (UTeM), Malaysia in 2013. Currently, he is pursuing a doctorate degree in Telecommunication Engineering from the Faculty of Engineering, University of Malaya, Kuala Lumpur, Malaysia. His research interests mainly include telecommunications, wireless networks, cloud networks, green communication, game theory, UAV, machine \& deep learning, IoT, and WSN.

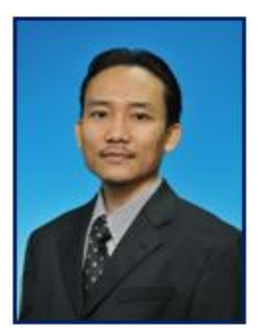

KAMARUL ARIFFIN BIN NOORDIN completed B. Eng. (Hons.) and M. Eng (Sc) from University of Malaya, Kuala Lumpur, Malaysia in 1998 and 2001, respectively, and $\mathrm{PhD}$ in Communication Systems from Lancaster University, the UK, in 2009. He is currently an associate professor in the Department of Electrical Engineering, University of Malaya, Kuala Lumpur, Malaysia. His research interests mainly include resource allocation in wireless networks, cognitive radio networks, device-to-device communications, network modelling, and performance analysis.

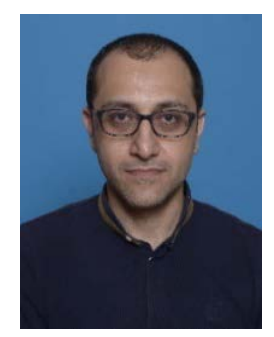

OMAR B. ABDULGHAFOOR completed his BSc in Control Engineering, M. Eng in Computer and Communication Engineering, and $\mathrm{PhD}$ in Telecommunication Engineering form the National University of Malaysia in 2015 (UKM). He is currently an assistant professor in the Department of Electronic and Telecommunication Engineering, American University of Kurdistan, Duhok, Iraq. His research interests include resource allocation in wireless networks, 5G cognitive radio networks, applications of game theory in wireless networks, and smart grid communications. 